\title{
LEUCOGRAMA E METABOLISMO OXIDATIVO DOS NEUTRÓFILOS DE CÃES COM LEISHMANIOSE VISCERAL ANTES E APÓS O TRATAMENTO COM ANTIMONIATO DE MEGLUMINA E ALOPURINOL
}

\author{
Paulo César Ciarlin ${ }^{1}$ Taiana Carvalho Valadares, ${ }^{2}$ Fabiana Augusta Ikeda-Garcia, ${ }^{3}$ \\ Mary Marcondes ${ }^{4}$ e Valéria Marçal Félix de Lima, ${ }^{4}$ \\ 1. Professor assistente, doutor, Departamento de Clínica, Cirurgia e Reprodução Animal, FMVA/UNESP. E-mail: ciarlini@fmva.unesp.br \\ 2. Graduanda em Medicina Veterinária, bolsista de Iniciação Científica da FAPESP, FMVA/UNESP \\ 3. Professora assitente, doutora, Departamento de Clínica, Cirurgia e Reprodução Animal, FMVA/UNESP \\ 4. Professora assistente, doutora, Departamento de Clínica, Cirurgia e reprodução Animal, FMVA/UNESP.
}

RESUMO

Compararam-se o leucograma e o metabolismo oxidativo dos neutrófilos de dez cães com leishmaniose visceral (LV) antes e após o início do tratamento com antimoniato de meglumina (AM) e com a associação de antimoniato de meglumina e alopurinol (AMA). Os resultados obtidos comprovaram que os neutrófilos de cães com LV não perdem sua capacidade de reduzir o tetrazólio nitroazul (NBT) e, na maioria dos casos, o metabolismo oxidativo apresentou-se mais ativo que o normal. Cães com LV apresentaram uma diminuição do metabolismo oxidativo dos neutrófilos após o tratamento com AM e AMA. O conjunto dos resultados sugere que a diminuição da taxa de redução do NBT está relacionada com a diminuição da carga parasitária de leishmania e a um possível efeito inibidor dessas drogas sobre o metabolismo oxidativo dos neutrófilos.

PALAVRAS-CHAVES: Função neutrofílica, glucantime, Leishmania sp., tetrazólio nitroazul (NBT).

ABSTRACT

\section{LEUKOGRAM AND NEUTROPHIL OXIDATIVE METABOLISM OF DOGS WITH VISCERAL LEISHMANIASIS BEFORE AND AFTER TREATMENT WITH MEGLUMINE ANTIMONIATE AND ALLOPURINOL}

Leukogram and neutrophil oxidative metabolism from dogs with visceral leishmaniasis (VL) were compared before and after treatment with meglumine antimoniate (AM) and with an association of meglumine antimoniate and allopurinol (AMA). The results obtained demonstrated that neutrophils of dogs with VL do not lose their capacity of reducing NBT and that oxidative metabolism has been more active in the majority of the cases. After treatment with AM and AMA, dogs with VL presented a redution of neutrophils oxidative metabolism, suggesting that this decrease was related with the decrease in the number of parasites and a probable inhibitor effect of these drugs on neutrophils oxidative metabolism.

KEYWORDS: Glucantime, Leishmania sp, nitroblue tetrazolium (NBT), neutrophil functions.

\section{INTRODUÇÃO}

A leishmaniose visceral (LV) canina tem como agentes etiológicos protozoários do gênero Leishma- nia, que, após penetrarem no organismo do hospedeiro vertebrado, replicam-se no interior de macrófagos, disseminando-se pelas vias hematógena e linfática (INIESTA et al., 2002). 
Apesar de haver inúmeras pesquisas sobre a patogenia da doença, especialmente direcionadas à resposta imune celular, poucos estudos enfocaram a função dos neutrófilos durante a infecção, em particular no que diz respeito ao seu metabolismo oxidativo. Os neutrófilos participam diretamente do controle da infecção na fase inicial da doença, porém parecem não exercer uma função antiparasitária significante em uma fase inicial (ROUSSEAU et al., 2001). Estudos realizados em modelos experimentais demonstraram que animais resistentes eliminam rapidamente o parasita logo no início da infecção, devido à participação de um grande número de neutrófilos que, mesmo sendo capazes de internalizar formas promastigotas de Leishmania sp., não são consideradas células hospedeiras, pois o parasita não se multiplica no interior desses polimorfonucleares (SMELT et al., 2000; AGA et al., 2002). A leucocitose tem sido associada à melhora clínica de cães portadores de leishmaniose (BOURDOISEAU et al., 1997), sendo a monocitose um achado comum, muitas vezes acompanhado da presença de grandes monócitos ativados, alguns dos quais se encontram parasitados (BURACCO et al., 1997; IKEDA et al., 2003).

Os neutrófilos interagem com os macrófagos (RIBEIRO-GOMES, 2007) e possuem função crítica na regulação do balanço das citocinas presentes no local da infecção, contribuindo para o desenvolvimento da imunidade específica e morte da Leishmania donovani na fase inicial da infecção (MCFARLANE et al., 2008). Além disso, em modelos experimentais a depleção de neutrófilos está associada a um aumento da carga parasitária de $L$. donovani no baço e no fígado (SMELT et al., 2000).

Todos os mecanismos que afetam o metabolismo oxidativo dos neutrófilos ainda não estão totalmente esclarecidos, entretanto, sabe-se que a fosfatase ácida isolada da superfície de promastigotas de L. donovani inibe a produção de superóxido de neutrófilos em seres humanos (SAHA et al., 1985). De forma semelhante, cães infectados por Leishmania sp. apresentam uma diminuição significativa no metabolismo oxidativo de neutrófilos (BRANDONISIO et al., 1996; VUOTTO et al., 2000). Quando o metabolismo oxidativo está diminuído, a Leishmania major pode sobreviver no interior de neutrófilos e a ativação desse metabolismo associa-se à eliminação intracelular do parasito (LAUFS et al., 2002).
O protocolo mais utilizado no tratamento de seres humanos com LV é a administração do antimoniato de meglumina, que tem sua distribuição realizada exclusivamente pelo Ministério da Saúde do Brasil. O mesmo medicamento é utilizado nos países do Mediterrâneo para o tratamento de cães com a enfermidade, como protocolo único, ou em associação com o alopurinol. O exato mecanismo de ação dos antimoniais não é conhecido, no entanto, BERMAN et al. (1985) observaram que sua ação leishmanicida está associada à inibição da glicólise e do ciclo do ácido cítrico da Leishmania. É possível que este mecanismo possa também comprometer a via glicolítica dos neutrófilos, acarretando a inibição de seu metabolismo oxidativo. RAIS et al. (2000) observaram que ratos e seres humanos tratados com antimonial têm a produção de superóxido potencializada. Segundo esses autores, o aumento da produção de superóxido no neutrófilo, após o tratamento, está associado à diminuição da carga parasitária e consequentemente ao desaparecimento da ação inibidora do antígeno sobre o metabolismo oxidativo das células do hospedeiro. Em animais portadores de leishmaniose visceral o tempo de vida do neutrófilo é menor, entretanto, estudos indicam que o tratamento com antimoniato de meglumina aumenta o tempo de vida desses polimorfonucleares (GUARGA et al., 2002).

Diante do exposto, o presente estudo objetivou avaliar o leucograma e o metabolismo oxidativo do neutrófilo de cães portadores de LV, antes e após o tratamento com antimoniato de meglumina e alopurinol.

\section{MATERIAL E METODOS}

Após aprovação da pesquisa junto ao Comitê de Ética Animal (Parecer 18/02 FOA-UNESP), selecionaram-se doze cães comprovadamente positivos para leishmaniose visceral. A leishmaniose foi confirmada mediante exames citológicos de punção aspirativa de linfonodo e medula óssea e sorologia utilizando o método de ensaio imunoenzimático (ELISA). A fim de evitar riscos de contaminação a outros animais ou a seres humanos, todos os cães utilizaram coleira antiparasitária à base de deltametrina (Scalibor ${ }^{\circledR}$, Intervet Production) e foram mantidos em um canil fechado e telado. 
Os cães foram aleatoriamente divididos em dois grupos. Tratou-se o primeiro grupo (AMA), formado por seis cães, com uma combinação do antimoniato de meglumina (Glucantime ${ }^{\circledR}$, Aventis Pharma) na dose de $75 \mathrm{mg} / \mathrm{kg}$, por via subcutânea, a cada doze horas por um período de 21 dias, associado ao alopurinol (Aluporinol, Hexal AG) na dose de $10 \mathrm{mg} / \mathrm{kg}$, por via oral, a cada doze horas durante três meses. O segundo grupo (AM), também formado por seis cães, foi tratado com antimoniato de meglumina na dose de $75 \mathrm{mg} / \mathrm{kg}$, por via subcutânea, a cada doze horas, durante 21 dias. Colheram-se amostras sanguíneas de todos os cães em três momentos diferentes: M1 (antes do tratamento), M2 (trinta dias após o início do tratamento) e M3 (sessenta dias após o início do tratamento).

De cada animal foram colhidos $10 \mathrm{~mL}$ de sangue, utilizando-se agulhas hipodérmicas e seringas descartáveis. Acondicionaram-se um volume de 0,5 $\mathrm{mL}$ em tubos plásticos estéreis contendo $10 \mathrm{U}$ de heparina sódica (liquemine ${ }^{\circledR}$ ) para realização do teste de redução do NBT e um volume de $4,5 \mathrm{~mL}$ de sangue em frascos de vidro contendo $5 \mathrm{mg}$ de EDTA-sódico para realização do leucograma. Empregaram-se $5 \mathrm{~mL}$ restantes para obtenção de soro para o teste sorológico da leishmaniose. As amostras sanguíneas foram mantidas refrigeradas até o momento do processamento laboratorial (até duas horas pós-colheita).

Procedeu-se também à punção da medula óssea na crista ilíaca dos animais. A punção aspirativa foi realizada ainda no linfonodo poplíteo ou no que apresentou aumento maior de volume.

Para pesquisa de formas amastigotas de Leishmania sp. no material colhido da medula óssea (crista ilíaca) e linfonodo, realizaram-se esfregaços corados com o corante hematológico panótico rápido (InstantProv, Newprov). Para a observação da presença de anticorpos no soro contra a Leishmania sp., foi utilizado o ELISA, conforme preconizado por LIMA et al. (2001).

Os testes da redução espontânea e estimulada do NBT foram realizados conforme descrito por CIARLINI et al. (2004). Para tal, fez-se uso de NBT tamponado (NBT- vial, SIGMA diagnostic) e estimulante composto por extrato bacteriano (Stimulant, SIGMA diagnostic). A porcentagem de células redutoras de NBT foi estabelecida a partir da contagem de 100 neutrófilos.
A contagem total de leucócitos foi realizada com auxílio de contador automático eletrônico de células sanguíneas (CC530 vet, CELM) e a contagem diferencial leucócitos foi feita em esfregaços sanguíneos tingidos com corante hematológico panótico rápido (Instant-Prov, Newprov), de acordo com as recomendações do fabricante e critérios de LASSEN \& WEISER (2004).

Ao término do experimento, seguindo as recomendações do Ministério da Saúde, todos os cães foram eutanasiados com pentobarbital sódico $(15 \mathrm{mg} / \mathrm{kg}$ por via intravenosa) seguido de uma ampola de $10 \mathrm{~mL}$ de cloreto de potássio a $19,1 \%$.

Utilizando-se um programa estatístico computadorizado(GraphPad InStat, v.3.05), após os estudos das distribuições de todas as variáveis quanto à normalidade (teste Kolmogorov-Smirnov) e homocedasticidade (teste Bartlett), aplicaram-se o teste de Friedman e o pós-teste de Dunn para as comparações entre os momentos experimentais.

\section{RESULTADOS E DISCUSSÃO}

Dois cães do grupo AM foram excluídos e submetidos à eutanásia, por apresentarem pneumopatia grave, aparentemente não associada à $\mathrm{LV}$ ou à medicação. A avaliação clínica e laboratorial dos cães do presente estudo foi recentemente descrita (IKEDAGARCIA et al., 2007), revelando que o tratamento promoveu remissão dos sinais clínicos e o retorno da ureia e da albumina sérica para os níveis normais, embora tenha promovido uma transitória hepatotoxidade.

Não obstante já relatado in vitro (LAUSF et al., 2002) e in vivo (IKEDA et al., 2003), no presente estudo não se observou qualquer forma amastigota de Leishmania sp. internalizada nos neutrófilos, concordando com as afirmações de SMELT et al. (2000) e AGA et al. (2002), de que o parasita não se multiplica dentro desses polimorfonucleares.

Antes do início do tratamento (M1) a contagem leucocitária dos dois grupos (AMA e AM) ficou dentro dos valores de normalidade considerados por MEINKOTH \& CLINKENBEARD (2000) para espécie canina (Tabelas 1 e 2). A ausência de alterações leucocitárias marcantes nos cães com leishmaniose utilizados neste estudo confirma relatos anteriores (IKEDA et al., 2003), de que o leucograma frequente- 
mente não se altera na LVC. Entretanto, a ausência de taxas elevadas de monócitos antes do tratamento difere de estudo anterior realizado em Araçatuba, SP, onde a monocitose ocorreu em 33,5\% dos cães portadores de LVC (IKEDA et al., 2003). Esse achado se contrapõe ainda às afirmações de BURACCO et al. (1997), de que cães com leishmaniose comumente apresentam aumento do número de monócitos circulantes. Tais divergências entre autores quanto ao leucograma de cães com LV expressam provavelmente diferenças quanto às fases da infecção, relação parasito-hospedeiro, assim como são reflexo de possíveis coinfecções não diagnosticadas nos diferentes estudos.

Verificou-se que o leucograma dos cães com LV submetidos a tratamento com antimonial e alopurinol não apresentou diferenças significativas entre o momento inicial, trinta e sessenta dias pós-tratamento (Tabela 1). Este resultado contraria a hipótese de BOURDOISEAU et al. (1997), de que a leucocitose está associada com a melhora clínica de cães portadores de leishmaniose.
Diferentemente, trinta dias após o tratamento com AM observou-se um aumento significativo da contagem total de leucócitos, neutrófilos e monócitos (Tabela 2). A elevação do número de neutrófilos em cães tratados com AM também foi observada por GUARDA et al. (2002), fortalecendo a hipótese de que o tratamento com antimoniato de meglumina aumenta o tempo de vida desses polimorfonucleares. Entretanto, após sessenta dias o quadro de neutrofília e monocitose não mais foi observado, sugerindo ser esta variação leucocitária uma resposta transitória associada à fase inicial de maior destruição da Leishmania sp. Além de o neutrófilo atuar diretamente e de modo significativo no controle da infecção na fase inicial da leishmaniose (ROUSSEAU et al., 2001; RIBEIRO-GOMES, 2007; MCFARLANE et al., 2008), a neutrofilia observada apenas nos primeiros trinta dias pós-tratamento sugere que essas células possam ter também um importante papel na resposta inespecífica durante a fase inicial dos tratamentos leishmanicidas.

TABELA 1. Valores médios (x) e desvios-padrão (s) do leucograma e da porcentagem (\%) de neutrófilos redutores do NBT na prova não estimulada (NE) e estimulada (E) de cães portadores da leishmaniose visceral, antes do tratamento com antimoniato de meglumina associado ao alopurinol (M1), trinta (M2) e sessenta (M3) dias após

\begin{tabular}{|c|c|c|c|}
\hline & M 1 & M2- & M3 \\
\hline & $\mathrm{x} \pm \mathrm{s}$ & $\mathrm{x} \pm \mathrm{s}$ & $\mathrm{x} \pm \mathrm{s}$ \\
\hline NBT-NE (\%) & $21,4 \pm 25,39^{a}$ & $2,2 \pm 2,17^{b}$ & $5,0 \pm 4,06^{\mathrm{ab}}$ \\
\hline NBT-E (\%) & $28,8 \pm 12,78^{\text {a }}$ & $9,0 \pm 5,48^{a b}$ & $6,6 \pm 6,58^{b}$ \\
\hline Leucócitos $\left(\times 10^{9} / \mathrm{L}\right)$ & $8,96 \pm 2,91^{\mathrm{a}}$ & $10,68 \pm 2,91^{\mathrm{a}}$ & $9,72 \pm 2,44^{\mathrm{a}}$ \\
\hline Segmentados $\left(\times 10^{9} / \mathrm{L}\right)$ & $5,05 \pm 2,59^{a}$ & $6,11 \pm 2,43^{a}$ & $5,51 \pm 2,86^{\mathrm{a}}$ \\
\hline Linfócitos $\left(\times 10^{9} / \mathrm{L}\right)$ & $2,59 \pm 1,01^{\mathrm{a}}$ & $2,81 \pm 1,31^{\mathrm{a}}$ & $3,00 \pm 2,03^{a}$ \\
\hline Monócitos $\left(\times 10^{9} / \mathrm{L}\right)$ & $0,58 \pm 0,49^{a}$ & $0,99 \pm 0,54^{\mathrm{a}}$ & $0,57 \pm 0,27^{\mathrm{a}}$ \\
\hline Eosinófilos $\left(\times 10^{9} / \mathrm{L}\right)$ & $0,58 \pm 0,24^{a}$ & $0,48 \pm 0,42^{\mathrm{a}}$ & $0,69 \pm 0,52^{\mathrm{a}}$ \\
\hline
\end{tabular}

*Letras não coincidentes na mesma linha indicam diferença significante $(\mathrm{P}<0,05)$.

Antes de receberem o tratamento com AMA ou AM, respectivamente $40 \%$ e $50 \%$ dos cães portadores de leishmaniose (Tabelas 1 e 2) apresentaram taxas neutrofílicas de redução espontânea do NBT (prova não estimulada) superiores às consideradas normais para a espécie como descrito por CIARLINI et al. (2004). No momento inicial, após serem estimulados, $80 \%$ dos cães do grupo AMA e 75\% do grupo AM apresentaram aumento nas taxas de redução de NBT (Tabelas 1 e 2).
A associação do aumento do metabolismo oxidativo dos neutrófilos com a Leishmania sp. também foi observada in vitro (LAUFS et al., 2002) e sugere que a inibição da produção de superóxido dos neutrófilos humanos, causada pela fosfatase ácida da membrana da forma promastigota da L. donovani (SAHA et al., 1985), não ocorre na LVC. Esses resultados também se contrapõem às observações de BRANDONISIO et al. (1996) e VUOTTO et al. (2000), que verificaram 
uma significante diminuição no metabolismo oxidativo em neutrófilos de cães naturalmente infectados pela Leishmania sp. Deve-se ressaltar que diferentemente do método citoquímico adotado no presente estudo, BRANDONISIO et al. (1996) e VUOTTO et al. (2000) utilizaram o método de quantificação de superóxido por citocromo c e quimiluminescência, respectivamente. Trata-se de métodos que requerem o isolamento dos neutrófilos do sangue total e, portanto, alteram os receptores de membrana responsáveis pela ativação do metabolismo oxidativo, além de tornarem a célula isolada isenta da influência de importantes componentes celulares e plasmáticos contidos no sangue total de cães com LV. Considerando que os animais deste experimento não apresentavam sinais de infecções secundárias, pode-se supor que a elevada porcentagem de redução do NBT observada antes do tratamento deveuse ao aumento do metabolismo oxidativo do neutrófilo induzido pela presença do parasito. Os resultados do presente estudo demonstram que o neutrófilo de cães com LV possui um metabolismo oxidativo funcional capaz de produzir grandes quantidades de superóxido e reduzir o NBT, sugerindo possivelmente que a capacidade imunossupressora da Leishmania sp. está associada a outros mecanismos.

TABELA 2. Valores médios (x) e desvios-padrão (s) do leucograma e da porcentagem (\%) de neutrófilos redutores do NBT na prova não estimulada (NE) e estimulada (E) de cães portadores da leishmaniose visceral, antes do tratamento com antimoniato de meglumina (M1), trinta (M2) e sessenta (M3) dias após

\begin{tabular}{lccc}
\hline & M1 & M2 & M3 \\
\cline { 2 - 4 } & $\mathrm{x} \pm \mathrm{s}$ & $\mathrm{x} \pm \mathrm{s}$ & $\mathrm{x} \pm \mathrm{s}$ \\
\hline NBT-NE (\%) & $10,3 \pm 9,61^{\mathrm{a}}$ & $2,3 \pm 2,63^{\mathrm{a}}$ & $1,8 \pm 2,22^{\mathrm{a}}$ \\
NBT-E $(\%)$ & $16,3 \pm 12,61^{\mathrm{a}}$ & $8,8 \pm 13,6^{\mathrm{a}}$ & $3,3 \pm 2,99^{\mathrm{a}}$ \\
Leucócitos $\left(\times 10^{9} / \mathrm{L}\right)$ & $6,5 \pm 0,82^{\mathrm{a}}$ & $20,5 \pm 13,1^{\mathrm{b}}$ & $9,2 \pm 2,07^{\mathrm{ab}}$ \\
Segmentados $\left(\times 10^{9} / \mathrm{L}\right)$ & $4,9 \pm 0,85^{\mathrm{a}}$ & $14,2 \pm 9,47^{\mathrm{b}}$ & $5,5 \pm 2,39^{\mathrm{ab}}$ \\
Linfócitos $\left(\times 10^{9} / \mathrm{L}\right)$ & $1,1 \pm 0.17^{\mathrm{a}}$ & $2,8 \pm 0,58^{\mathrm{a}}$ & $2,5 \pm 0,58^{\mathrm{a}}$ \\
Monócitos $\left(\times 10^{9} / \mathrm{L}\right)$ & $0,2 \pm 0,07^{\mathrm{a}}$ & $1,8 \pm 1,78^{\mathrm{b}}$ & $0,5 \pm 0,15^{\mathrm{ab}}$ \\
Eosinófilos $\left(\times 10^{9} / \mathrm{L}\right)$ & $0.2 \pm 0.22^{\mathrm{a}}$ & $0.7 \pm 1.30^{\mathrm{a}}$ & $0.6 \pm 0.40^{\mathrm{a}}$ \\
\hline
\end{tabular}

*Letras não coincidentes na mesma linha indicam diferença significante $(\mathrm{P}<0,05)$.

RAIS et al. (2000) observaram que o uso de droga antimoniais em seres humanos e ratos causa um aumento do metabolismo oxidativo dos neutrófilos devido à diminuição da carga parasitária. Entretanto, no presente estudo, após trinta e sessenta dias de tratamento com AM e AMA, nenhum animal apresentou valores elevados de redução do NBT na prova não estimulada, e as menores taxas de neutrófilos redutores de NBT coincidiram com a melhora clínica e a diminuição da carga parasitária dos cães pós-tratamentos (Tabelas $1 \mathrm{e}$ 2). Há evidências de que a capacidade de o neutrófilo reduzir o NBT após o tratamento está relacionada ainda com outros mecanismos, além da diminuição da carga parasitária. Trinta e sessenta dias após ter iniciado o tratamento com AM e AMA, respectivamente, 25\% $75 \%$ e $20 \% 40 \%$ dos cães apresentaram taxas de redução do NBT espontânea (NBT-NE) inferiores aos valores de normalidade considerados por CIARLINI et al. (2004). A capacidade de redução do NBT do neutrófilo estimulado (NBT-E) também diminuiu em ambos os tratamentos, observando-se menores valores após sessenta dias no grupo AM. Coincidentemente, 90\% dos animais apresentaram melhora clínica e tornaram-se assintomáticos após o tratamento com AM e AMA. Esses baixos valores de redução do NBT póstratamento sugerem um possível efeito inibidor da AM e AMA sobre o metabolismo do neutrófilo. É provável que a inibição da glicólise e do ciclo do acido cítrico do parasita causado pelos antimoniais (BERMAN et al., 1985) também possa comprometer a via glicolítica do neutrófilo, acarretando a inibição de seu metabolismo oxidativo. Os neutrófilos sofrem uma "explosão respiratória" quando ativados, ocasionando um grande consumo de glicose e oxigênio (CIARLINI et al., 2001), de modo que a ação inibidora dos antimoniais sobre a via glicolítica neutrofílica pode diminuir a produção 
de superóxido e consequentemente a taxa de redução do NBT, conforme verificado no presente estudo.

Considerando que os tratamentos adotados contribuíram para uma melhora clínica, porém, não para uma total eliminação da infecção, torna-se necessário investigar se a limitação dos protocolos atualmente preconizados para o tratamento da LVC está associada a um possível efeito inibidor da AM e AMA sobre o metabolismo oxidativo do neutrófilo.

\section{CONCLUSÃO}

Neutrófilos de cães com leishmaniose visceral possuem um metabolismo oxidativo funcional capaz de produzir grandes quantidades de superóxido e reduzir o NBT.

O tratamento com antimoniato de meglumina e alopurinol em cães naturalmente infectados com Leishmania sp. promove uma diminuição do metabolismo oxidativo do neutrófilos que coincide com a melhora clínica e diminuição da carga parasitária.

\section{REFERÊNCIAS}

AGA, E.; KATSCHINSKI, D. M.; VAN ZANDBERGEN, G.; LAUFS, H. Inhibition of the spontaneous apoptosis of neutrophil granulocytes by the intracellular parasite Leishmania major. Journal of Immunology, v. 169, n. 2, p. 898-905, 2002.

BERMAN, J. D.; WADDELL, D.; HANSON, B. D. Biochemical mechanisms of the antileishmanial activity of sodium stibogluconate. Antimicrobial Agents and Chemotherapy, v. 27, n. 6, p. 916-920, 1985.

BOURDOISEAU, G.; BONNEFONT, C.; CHABANNE, L. Modifications sanguines (cellulaires et humorales) chez le chien leishmanien suivi de chiens infectés traités et non traités. Veterinary Immunology and Immunopathology, v. 148, n. 3, p. 219-228, 1997.

BRANDONISIO, O.; PANUNZIO, M.; FALIERO, S. M.; CECI, L.; FASANELLA, A.; PUCCINI, V. Evaluation of polymorphonuclear cell and monocyte functions in Leishmania infanctum infected dogs. Veterinary Immunology and Immunopathology, v. 53, n. 1-2, p. 95-103, 1996.

BURACCO, P.; ABATE, O.; GUGLIELMINO, R.; MORELLO, E. Osteomyelitis and arthrosynovitis associated whit Leishmania donovani infection in a dog. Journal of Small Animal Practice, v. 38, n. 1, p. 29-30, 1997.
CIARLINI, P. C.; CIARLINI, L. D. R. P.; KOHAYAGAWA, A.; TAKAHIRA, R. K.; LEINZ, F. F. Metabolismo oxidativo de neutrófilos em ovelhas naturalmente infectadas por nematódeos gastrintestinais e suas correlações com níveis séricos de progesterona e a carga parasitária. Revista Brasileira de Medicina Veterinária, v. 23 , n. 4, 2001. p. 156-159.

CIARLINI, P. C.; PATRÍCIO, R. F.; COUTO, R.; BONELLO, F. L. Efeito da vacina polivalente sobre o leucograma e o metabolismo oxidativo dos neutrófilos em cães. Arquivo do Instituto Biológico, Brasil, v. 71, n. 3, p. 323-327, 2004.

GUARGA, J. L.; MARENO, J.; LUCIENTES, J.; GARCIA, M. J.; ANGEL, P. M. Evaluation of aspecific immunochemotherapy for the treatment of canine visceral leishmaniasis. Veterinary Immunology and Immunopathology, v. 88, n. 1-2, p. 13-20, 2002.

IKEDA, F. A.; CIARLINI, P. C.; FEITOSA, M. M.; GONÇALVES, M. E.; LUVIZOTTO, M. C. R.; LIMA, V. M. F. Perfil hematológico de cães naturalmente infectados por Leishmania chagasi no município de Araçatuba - SP: um estudo retrospectivo de 191 casos. Clínica Veterinária, n. 47, p. 42-48, 2003.

IKEDA-GARCIA, F. A.; LOPES, P. C.; CIARLINI, P. C.; MARQUES, F. J.; LIMA, V. M. F.; PERRI, S. H. V.; FEITOSA, M. M. Evaluation of renal and hepatic functions in dogs naturally infected by visceral leishmaniasis submitted to treatment with meglumine antimoniate. Research in Veterinary Science, v. 83, p. 105-108, 2007.

INIESTA, V.; NIETO, L. C. G.; MOLANO, I.; MOHEDANO, A. Arginase I induction in macrophages, triggered by Th2-type cytokines, supports the growth of intracellular Leishmania parasites. Parasite Immunology, v. 24, p. 113-118, 2002.

LASSEN, E. D.; WEISER, G. Laboratory technology for veterinary medicine. In: THRALL, M. A.; BAKER, D. C.; CAMPBELL, T. W.; DENICOLA, D.; FETTMAN, M. J.; LASSEN, E. D.; REBAR, A.; WEISER, G. Veterinary hematology and clinical chemistry. Philadelphia: Lippincott Williams \& Wilkins, 2004. p. 3-37.

LAUFS, H.; MÜLLER, K.; FLEISCHER, J.; REILING, N.; JAHNKE, N.; JENSENIUS, W. S.; LASKAY, T. Intracellular survival of Leishmania major in neutrophil granulocytes after uptake in the absence of heat-labile serum factors. Infection and Immunity, v. 70, n. 2, p. 826-835, 2002.

LIMA, V. M. F.; CÂNDIDO, T. C.; MOREIRA, M. B.; JUNIOR, F.; BIDO, A. V.; NETO, J. L. S.; LUVIZZOTO, M. R. Evaluation of an ELISA assay for the serodiagnosis of canine visceral leishmaniasis compared to indirect immunofluorescence assay and cytology. Revista da Sociedade Brasileira de Medicina Tropical, v. 34, supl. II, p. 176, 2001. 
McFARLANE, E.; PEREZ, C.; CHARMOY, M.; ALLENBACH, C.; CARTER, K. C.; ALEXANDER, J.; TACCHINI-COTTIER,

F. Neutrophil contribui to development of a protective immune response during onset of infection with Leishmania donovani. Infection and Immunity, v. 76, n. 2, p. 532-541, 2008.

MEINKOTH, J. H.; CLINKENBEARD, K. D. Normal hematology of the dog. In: FELDMAN, B. F.; ZINKL, J. G.; JAIN, N. C. Schalm's veterinary hematology. 5. ed. Philadelphia: Lippincott Willians and Wilkins, 2000. p. 1057-1063.

RAIS, S.; PERIANIN, A.; LENOIR, M.; SADAK; A.; RIVOLLET, D.; PAUL, M.; DENIAU, M. Sodium stibogluconate (pentostam) potentiates oxidant production in murine visceral leishmaniasis and in human blood. Antimicrobial Agents and Chemotherapy, v. 44, n. 9, p. 2406-2410, 2000.

RIBEIRO-GOMES, F. L. Neutrophils activate macrophages for intracellular killing of Leishmania major through recruitment of TLR4 by neutrophil elastase. The Journal of Immunology, v. 179, p. 3988-3994, 2007.
ROUSSEAU, D. In vivo involvement of polymorphonuclear neutrophils in Leishmania infantum infection. BMC Microbiology, on-line, v. 1, n.17, 2001. Disponível em: $<$ http://www.biomedcentral.com/1471-2180/1/17.> Acesso em: 20 set. 2008.

SAHA, A.K.; DAS, S.; GLEW, R.H.; GOTTLIEB, M. Resistance of Leishmanial Phosphatases to Inactivation by Oxygen Metabolites. Journal of Clinical Microbiology, v. 22, n. 3, p. 329-332, 1985.

SMELT, S. C.; COTTERELL, S. E. J.; ENGWERDA, C. R.; KAYE, P. M. B cell-deficient mice are highly resistant to Leishmania donovani infection, but develop neutrophil-mediated tissue pathology. Journal of Immunology, v. 164, p. 3681-3688, 2000.

VUOTTO, M. L.; DE LUNA, R.; IELPO, M. T. L.; DE-SOLE, P.; MOSCATIELLO, V.; CIMEONE, I. Chemiluminescence activity in whole blood phagocytes of dogs naturally infected with Leishmania infantum. Luminescence Chichester, v. 15, n. 4, p. 251-255, 2000.

Protocolado em: 30 out. 2007. Aceito em: 18 jan. 2010. 